\title{
CONVERGENCE OF AN ITERATIVE ALGORITHM FOR TEICHMÜLLER MAPS VIA HARMONIC ENERGY OPTIMIZATION
}

\author{
LOK MING LUI, XIANFENG GU, AND SHING-TUNG YAU
}

\begin{abstract}
Finding surface mappings with least distortion arises from many applications in various fields. Extremal Teichmüller maps are surface mappings with least conformality distortion. The existence and uniqueness of the extremal Teichmüller map between Riemann surfaces of finite type are theoretically guaranteed (see Fletcher and Markovic, Quasiconformal maps and Teichmüller theory, Oxford Graduate Texts in Math., vol. 11, Oxford University Press, Oxford, 2007). Recently, a simple iterative algorithm for computing the Teichmüller maps between connected Riemann surfaces with given boundary value was proposed by Lui, Lam, Yau, and Gu in Teichmüller extremal mapping and its applications to landmark matching registration, arXiv:1211.2569. Numerical results were reported in the paper to show the effectiveness of the algorithm. The method was successfully applied to landmarkmatching registration. The purpose of this paper is to prove the iterative algorithm proposed in loc. cit., indeed converges.
\end{abstract}

\section{INTRODUCTION}

Finding meaningful surface mappings with least distortion has fundamental importance. Applications can be found in different areas such as registration, shape analysis and grid generation. Conformal mapping has been widely used to establish a good one-to-one correspondence between different surfaces, since it preserves the local geometry well $2[18$. The Riemann mapping theorem guarantees the existence of conformal mappings between simply-connected surfaces. However, this fact is not valid for general Riemann surfaces. Given two Riemann surfaces with different conformal modules, there is generally no conformal mapping between them. In this case, it is usually desirable to obtain a mapping that minimizes the conformality distortion. Every diffeomorphic surface mapping is associated with a unique Beltrami differential, which is a complex-valued function, $\mu_{f}$, defined on the source surface. The Beltrami differential, $\mu_{f}$, measures the deviation of the mapping from a conformal map. Given two Riemann surfaces $S_{1}$ and $S_{2}$, there exists a unique and bijective map $f: S_{1} \rightarrow S_{2}$, called the Teichmüller map, minimizing the $L^{\infty}$ norm of the Beltrami differential [1]. Therefore, the extremal Teichmüller map can be considered as the 'most conformal' map between Riemann surfaces of the same topology, which is a natural extension of conformal mappings.

Received by the editor September 18, 2013.

2010 Mathematics Subject Classification. Primary 52C26, 65D18, 65E05; Secondary 52B20, $52 \mathrm{C} 99$.

The first author was supported by RGC GRF (Project ID: 401811), CUHK Direct Grant (Project ID: 2060413), and CUHK FIS Grant (Project ID: 1902036). 
1.1. Extremal problem. Mathematically, the extremal problem for obtaining a surface mapping with least conformality distortion can be formulated as follows. Suppose $\left(S_{1}, \sigma|d z|^{2}\right)$ and $\left(S_{2}, \rho|d w|^{2}\right)$ are two Riemann surfaces of finite type, where $z$ and $w$ are their conformal parameters respectively. Every diffeomorphism between $S_{1}$ and $S_{2}$ is associated with a unique Beltrami differential. A Beltrami differential $\mu(z) \frac{d \bar{z}}{d z}$ on the Riemann surface $S_{1}$ is an assignment to each chart $\left(U_{\alpha}, \phi_{\alpha}\right)$ of an $L^{\infty}$ complex-valued function $\mu_{\alpha}$, defined on the local parameter $z_{\alpha}$. Then, $f: S_{1} \rightarrow S_{2}$ is said to be a quasi-conformal mapping associated with the Beltrami differential $\mu(z) \frac{\overline{d z}}{d z}$ if for any chart $\left(U_{\alpha}, \phi_{\alpha}\right)$ on $S_{1}$ and any chart $\left(V_{\beta}, \psi_{\beta}\right)$ on $S_{2}$, the mapping $f_{\alpha \beta}:=\psi_{\beta} \circ f \circ \phi_{\alpha}^{-1}$ is quasi-conformal associated with $\mu_{\alpha}\left(z_{\alpha}\right) \frac{d \overline{z_{\alpha}}}{d z_{\alpha}}$.

Our goal is to look for an extremal quasi-conformal mapping, which is extremal in the sense of minimizing the $\|\cdot\|_{\infty}$ over all Beltrami differentials corresponding to quasi-conformal mappings between $S_{1}$ and $S_{2}$. The idea of extremality is to make the supreme norm of the Beltrami differential as small as possible such that $f$ is as 'nearly conformal' as possible.

The extremal problem can therefore be formulated as finding $f: S_{1} \rightarrow S_{2}$ that solves:

$$
f=\operatorname{argmin}_{g \in \mathcal{A}}\left\{\left\|\mu_{g}\right\|_{\infty}\right\},
$$

where $\mathcal{A}=\left\{g: S_{1} \rightarrow S_{2}: g\right.$ is a diffeomorphism $\}$.

The above optimization problem (1.1) has a unique global minimizer provided that $S_{1}$ and $S_{2}$ are Riemann surfaces of finite type. Also, the unique minimizer $f: S_{1} \rightarrow S_{2}$ is a Teichmüller map, that is, its associated Beltrami differential $\mu_{f}$ is of the following form:

$$
\mu_{f}=k \frac{\bar{\varphi}}{|\varphi|}
$$

where $0 \leq k<1$ is a non-negative real constant and $\varphi$ is an integrable holomorphic function defined on $S_{1}(\varphi \neq 0)$. Beltrami differential of this form is said to be of Teichmüller type.

1.2. An iterative algorithm for Teichmüller maps. To solve the extremal problem (1.1) to obtain the Teichmüller map between connected surfaces, an iterative algorithm was proposed in 20, called the quasi-conformal (QC) iteration. The ultimate goal is to obtain the extremal map between connected (either simplyconnected or multiply-connected) surfaces with given boundary value, which minimizes the conformality distortion. The basic idea of the iterative algorithm is to project the Beltrami differential to the space of all Beltrami differentials of constant modulus, and compute a quasi-conformal map whose Beltrami differential is closest to the projection in the least square sense. More specifically, the QC iteration for solving (1.1) can be described as follows:

$$
\left\{\begin{array}{l}
f_{n+1}=\mathbf{L B S}\left(\mu_{n+1}\right), \\
\widetilde{\mu}_{n+1}=\mu_{n}+\alpha \mu\left(f_{n+1}, \mu_{n}\right), \\
\mu_{n+1}=\mathcal{L}\left(\mathcal{P}\left(\widetilde{\mu}_{n+1}\right)\right),
\end{array}\right.
$$

where $f_{n}$ is the quasi-conformal map obtained at the $n^{\text {th }}$ iteration, $\nu_{n}$ is the Beltrami differential of $f_{n}$ and $\mu_{n}$ is a Beltrami differential of constant modulus. 
$\operatorname{LBS}(\mu)$ is the operator to obtain a quasi-conformal mapping whose Beltrami differential is closest to $\mu$ in the least square sense. In other words,

$$
\mathbf{L B S}(\mu)=\operatorname{argmin}_{f \in \mathcal{A}}\left\{\int_{S_{1}}\left|\frac{\partial f}{\partial \bar{z}}-\mu \frac{\partial f}{\partial z}\right|^{2} d S_{1}\right\} .
$$

$\mu\left(f_{n+1}, \nu_{n}\right)$ denotes the Beltrami differential of $f_{n+1}$ under the auxiliary metric with respect to $\nu_{n}$, namely, $\left|d z+\nu_{n} d \bar{z}\right|^{2}\left(|d z|^{2}\right.$ is the original metric on $\left.S_{1}\right)$. More precisely, $\mu\left(f_{n+1}, \nu_{n}\right)$ can be explicitly computed as follows:

$$
\mu\left(f_{n+1}, \nu_{n}\right)=\left(\frac{\partial f_{n+1}}{\partial \bar{z}}+\nu_{n} \frac{\partial f_{n+1}}{\partial z}\right) /\left(\frac{\partial f_{n+1}}{\partial z}-\overline{\nu_{n}} \frac{\partial f_{n+1}}{\partial \bar{z}}\right) .
$$

$\mathcal{P}\left(\widetilde{\mu}_{n+1}\right)$ is the operator used to project $\widetilde{\mu}_{n+1}$ to the space of Beltrami differentials with constant modulus; it is defined as

$$
\mathcal{P}\left(\widetilde{\mu}_{n+1}\right)=\mu_{n}+\epsilon w_{n},
$$

where $w_{n}: S_{1} \rightarrow \mathbb{C}$ and $\epsilon: S_{1} \rightarrow \mathbb{R}^{+}$is a suitable real function on $S_{1}$ such that $\left|\mu_{n}+\epsilon w_{n}\right|$ is a constant.

In practice, the projection operator can be simplified as

$$
\mathcal{P}\left(\widetilde{\mu}_{n+1}\right)=\left(\frac{\int_{S_{1}}\left|\widetilde{\mu}_{n+1}\right| d S_{1}}{\int_{S_{1}} d S_{1}}\right) \frac{\widetilde{\mu}_{n+1}}{\left|\widetilde{\mu}_{n+1}\right|}
$$

$\mathcal{L}$ is the Laplacian smoothing operator to smooth out $\widetilde{\theta}_{n+1}:=\arg \left(\mathcal{P}\left(\widetilde{\mu}_{n+1}\right)\right)$ through

$$
\frac{d \widetilde{\theta}_{n+1}}{d t}=\Delta \widetilde{\theta}_{n+1}
$$

where $\widetilde{\theta}_{n+1}$ is smoothed out for a given time period $t>0$.

Both $\mathcal{L}\left(\mathcal{P}\left(\nu_{n}\right)\right), \mathbf{L B S}\left(\mu_{n+1}\right)$ and $\mu\left(f_{n+1}, \nu_{n}\right)$ can be easily computed. In particular, the discretization of $\mathbf{L B S}\left(\mu_{n+1}\right)$ on a triangulation mesh can be reduced to a least square problem of a linear system.

When $\mu\left(f_{n+1}, \mu_{n}\right)$ is small, $\alpha$ can be chosen to be 1 . Then, $\mu_{n}+\alpha \mu\left(f_{n+1}, \mu_{n}\right) \approx$ $\mu\left(f_{n+1}, 0\right)$, where $\mu\left(f_{n+1}, 0\right)$ is the Beltrami differential of $f_{n+1}$ under the original metric. The QC iteration can be further modified as

$$
\left\{\begin{array}{l}
f_{n+1}=\mathbf{L B S}\left(\mu_{n}\right), \\
\widetilde{\mu}_{n+1}=\mu\left(f_{n+1}, 0\right), \\
\mu_{n+1}=\mathcal{L}\left(\mathcal{P}\left(\widetilde{\mu}_{n+1}\right)\right) .
\end{array}\right.
$$

The QC iteration (1.3) is very efficient. Also, numerical results reported in 20] demonstrate that the proposed iteration can compute the Teichmüller map accurately, even on highly irregular meshes. The algorithm was successfully applied to landmark-based registration for applications in medical imaging and computer graphics.

This paper is to provide a complete analysis of the above iterative algorithm (1.3). In particular, we prove the convergence of (1.3) that $f_{n}$ and $\mu_{n}$ respectively converges to the extremal map $f^{*}$ and its associated Beltrami differential $\mu^{*}$, which solves the optimization problem (1.1).

We remark that although the iterative algorithm is designed for obtaining an extremal map between connected surfaces with given boundary values, the convergence proof applies to general Riemann surfaces of finite type (such as high-genus 


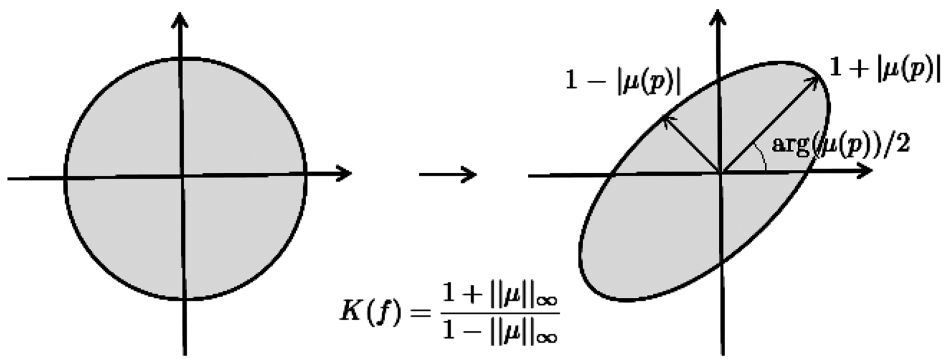

FiguRE 1. Illustration of how the Beltrami coefficient determines the conformality distortion.

closed surfaces). In other words, the QC iteration can be applied to computing extremal maps between general Riemann surfaces. For the ease of presentation, we will restrict our discussion to the situation when both $S_{1}$ and $S_{2}$ are either simply-connected or multiply-connected open surfaces.

1.3. Organization. This paper is organized as follows. In Section 2, we describe some mathematical background, which is relevant to this work. In Section 3, we reformulate the extremal problem defined by (1.1) as the optimization problem of the harmonic energy, which helps us to understand the iterative algorithm (1.3) better. In Section 4, we prove the convergence of the QC iteration to our desired extremal Teichmüller map. A concluding remark will be given in Section 6.

\section{MathematicAl BACKGROUND}

2.1. Quasi-conformal mappings and Beltrami equation. In this section, we describe some basic mathematical concepts relevant to our algorithms. For details, we refer the readers to [19,21.

A surface $S$ with a conformal structure is called a Riemann surface. Given two Riemann surfaces $S_{1}$ and $S_{2}$, a map $f: S_{1} \rightarrow S_{2}$ is conformal if it preserves the surface metric up to a multiplicative factor called the conformal factor. A generalization of conformal maps is the quasi-conformal maps, which are orientation preserving homeomorphisms between Riemann surfaces with bounded conformality distortion, in the sense that their first order approximations takes small circles to small ellipses of bounded eccentricity [19]. Mathematically, $f: \mathbb{C} \rightarrow \mathbb{C}$ is quasiconformal provided that it satisfies the Beltrami equation

$$
\frac{\partial f}{\partial \bar{z}}=\mu(z) \frac{\partial f}{\partial z}
$$

for some complex-valued function $\mu$ satisfying $\|\mu\|_{\infty}<1 . \mu$ is called the Beltrami coefficient, which is a measure of non-conformality. $\mu$ measures how far the map is deviated from a conformal map. $\mu \equiv 0$ if and only if $f$ is conformal. Infinitesimally, around a point $p, f$ may be expressed with respect to its local parameter as follows:

$$
\begin{aligned}
f(z) & =f(p)+f_{z}(p) z+f_{\bar{z}}(p) \bar{z} \\
& =f(p)+f_{z}(p)(z+\mu(p) \bar{z}) .
\end{aligned}
$$

Obviously, $f$ is not conformal if and only if $\mu(p) \neq 0$. Inside the local parameter domain, $f$ may be considered as a map composed of a translation to $f(p)$ together with a stretch map $S(z)=z+\mu(p) \bar{z}$, which is postcomposed by a multiplication 


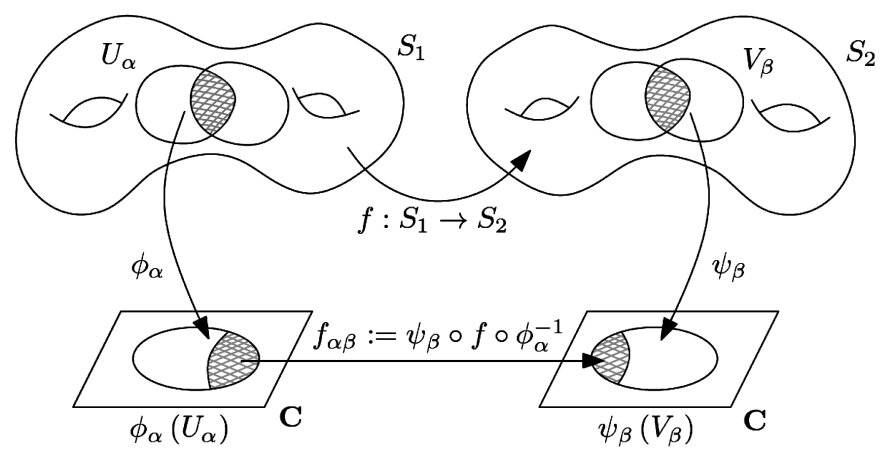

FIGURE 2. Illustration of the definition of quasi-conformal map between Riemann surfaces.

of $f_{z}(p)$. All the conformal distortion of $S(z)$ is caused by $\mu(p) . S(z)$ is the map that causes $f$ to map a small circle to a small ellipse. From $\mu(p)$, we can determine the directions of maximal magnification and shrinking and the amount of their distortions as well. Specifically, the angle of maximal magnification is $\arg (\mu(p)) / 2$ with magnifying factor $1+|\mu(p)|$. The angle of maximal shrinking is the orthogonal angle $(\arg (\mu(p))-\pi) / 2$ with shrinking factor $1-|\mu(p)|$. Thus, the Beltrami coefficient $\mu$ gives us all the information about the properties of the map (see Figure 1).

The maximal dilation of $f$ is given by

$$
K(f)=\frac{1+\|\mu\|_{\infty}}{1-\|\mu\|_{\infty}} .
$$

Quasiconformal mapping between two Riemann surfaces $S_{1}$ and $S_{2}$ can also be defined. Instead of the Beltrami coefficient, the Beltrami differential has to be used. A Beltrami differential $\mu(z) \frac{d \bar{z}}{d z}$ on the Riemann surface $S_{1}$ is an assignment to each chart $\left(U_{\alpha}, \phi_{\alpha}\right)$ of an $L^{\infty}$ complex-valued function $\mu_{\alpha}$, defined on local parameter $z_{\alpha}$ such that

$$
\mu_{\alpha}\left(z_{\alpha}\right) \frac{d \overline{z_{\alpha}}}{d z_{\alpha}}=\mu_{\beta}\left(z_{\beta}\right) \frac{d \overline{z_{\beta}}}{d z_{\beta}}
$$

on the domain which is also covered by another chart $\left(U_{\beta}, \phi_{\beta}\right)$, where $\frac{d z_{\beta}}{d z_{\alpha}}=\frac{d}{d z_{\alpha}} \phi_{\alpha \beta}$ and $\phi_{\alpha \beta}=\phi_{\beta} \circ \phi_{\alpha}^{-1}$.

An orientation preserving diffeomorphism $f: S_{1} \rightarrow S_{2}$ is called quasi-conformal associated with $\mu(z) \frac{\overline{d z}}{d z}$ if for any chart $\left(U_{\alpha}, \phi_{\alpha}\right)$ on $S_{1}$ and any chart $\left(V_{\beta}, \psi_{\beta}\right)$ on $S_{2}$, the mapping $f_{\alpha \beta}:=\psi_{\beta} \circ f \circ \phi_{\alpha}^{-1}$ is quasi-conformal associated with $\mu_{\alpha}\left(z_{\alpha}\right) \frac{d \overline{z_{\alpha}}}{d z_{\alpha}}$. See Figure 2 for an illustration.

2.2. Extremal maps and Teichmüller maps. A special class of quasi-conformal maps is called the extremal maps, which minimize the conformality distortion. More specifically, an extremal quasi-conformal map between $S_{1}$ and $S_{2}$ is extremal in the sense of minimizing the $\|\cdot\|_{\infty}$ over all Beltrami differentials corresponding to quasiconformal mappings between the two surfaces. An extremal map always exists but need not be unique. Mathematically, an extremal quasi-conformal mapping can be defined as follows: 
Definition 2.1. Suppose $S_{1}$ and $S_{2}$ are connected Riemann surfaces with boundaries. Let $f: S_{1} \rightarrow S_{2}$ be a quasi-conformal mapping between $S_{1}$ and $S_{2}$. $f$ is said to be an extremal map if for any quasi-conformal mapping $h: S_{1} \rightarrow S_{2}$ isotopic to $f$ relative to the boundary,

$$
K(f) \leq K(h) .
$$

It is uniquely extremal if the inequality (2.5) is strict whenever $f \neq h$.

Closely related to the extremal map is the Teichmüller map. It is defined as follows.

Definition 2.2. Let $f: S_{1} \rightarrow S_{2}$ be a quasi-conformal mapping. $f$ is said to be a Teichmüller map associated to the integrable holomorphic function $\varphi: S_{1} \rightarrow \mathbb{C}$ if its associated Beltrami differential is of the form

$$
\mu(f)=k \frac{\bar{\varphi}}{|\varphi|}
$$

for some constant $k<1$ and holomorphic function $\varphi \neq 0$ with $\|\varphi\|_{1}=\int_{S_{1}}|\varphi|<\infty$.

In other words, a Teichmüller map is a quasi-conformal mapping with uniform conformality distortion over the whole domain.

An extremal map might not be unique. However, a Teichmüller map associated with a holomorphic function is the unique extremal map in its homotopic class. In particular, a Teichmuller map between two connected open surfaces with suitable given boundary values is the unique extremal map. Strebel's theorem explains the relationship bewtween the Teichmüller map and an extremal map.

Definition 2.3 (Boundary dilation). The boundary dilation $K_{1}[f]$ of $f$ is defined as

$$
K_{1}[f]=\inf _{C}\left\{K\left(\left.h\right|_{S_{1} \backslash C}\right): h \in \mathfrak{F}, C \subseteq S_{1}, C \text { is compact }\right\} .
$$

where $\mathfrak{F}$ is the family of quasi-conformal mappings of $S_{1}$ onto $S_{2}$ which are homotopic to $f$ modulo the boundary.

Theorem 2.4 (Strebel's theorem; see [22], page 319). Let $f$ be an extremal quasiconformal map with $K(f)>1$. If $K_{1}[f]<K(f)$, then $f$ is a Teichmüller map associated with an integrable holomorphic function on $S_{1}$. Hence, $f$ is also a unique extremal map.

In other words, an extremal map between $S_{1}$ and $S_{2}$ with suitable boundary condition is a Teichmüller map. In particular, the Teichmüller map and extremal map of the unit disk are closely related.

Theorem 2.5 (See [23], page 110). Let $g: \partial \mathbb{D} \rightarrow \partial \mathbb{D}$ be an orientation-preserving homeomorphism of $\partial \mathbb{D}$. Suppose further that $h^{\prime}\left(e^{i \theta}\right) \neq 0$ and $h^{\prime \prime}\left(e^{i \theta}\right)$ is bounded. Then there is a Teichmüller map $f$ that is the unique extremal extension of $g$ to $\mathbb{D}$. That is, $f: \mathbb{D} \rightarrow \mathbb{D}$ is an extremal mapping with $\left.f\right|_{\partial \mathbb{D}}=g$.

Thus, if the boundary correspondence satisfies certain conditions on its derivatives, the extremal map of the unit disk must be a Teichmüller map.

Now, in the case when interior landmark constraints are further enforced, the existence of unique Teichmüller map can be guaranteed if the boundary and landmark correspondence satisfy suitable conditions. The unique Teichmüller map is 
extremal, which minimizes the maximal conformality distortion. The following theorem can be derived immediately from Strebel's Theorem (Theorem 2.4):

Theorem 2.6. Let $\left\{p_{i}\right\}_{i=1}^{n} \in S_{1}$ and $\left\{q_{i}\right\}_{i=1}^{n} \in S_{2}$ be the corresponding interior landmark constraints. Let $f: S_{1} \backslash\left\{p_{i}\right\}_{i=1}^{n} \rightarrow S_{2} \backslash\left\{q_{i}\right\}_{i=1}^{n}$ be the extremal map, such that $p_{i}$ corresponds to $q_{i}$ for all $1 \leq i \leq n$. If $K_{1}[f]<K(f)$, then $f$ is a Teichmüller map associated with an integrable holomorphic function on $S_{1} \backslash\left\{p_{i}\right\}_{i=1}^{n}$. Hence, $f$ is a unique extremal map.

In particular, a unique Teichmüller map $f: \mathbb{D} \rightarrow \mathbb{D}$ between unit disks with interior landmark constraints enforced exists, if the boundary map $\left.f\right|_{\partial \mathbb{D}}$ satisfies suitable conditions. The following theorem can be obtained directly from Theorem 2.5 .

Theorem 2.7. Let $g: \partial \mathbb{D} \rightarrow \partial \mathbb{D}$ be an orientation-preserving homeomorphism of $\partial \mathbb{D}$. Suppose further that $h^{\prime}\left(e^{i \theta}\right) \neq 0$ and $h^{\prime \prime}\left(e^{i \theta}\right)$ is bounded. Let $\left\{p_{i}\right\}_{i=1}^{n} \in \mathbb{D}$ and $\left\{q_{i}\right\}_{i=1}^{n} \in \mathbb{D}$ be the corresponding interior landmark constraints. Then there is a Teichmüller map $f: \mathbb{D} \backslash\left\{p_{i}\right\}_{i=1}^{n} \rightarrow \mathbb{D} \backslash\left\{q_{i}\right\}_{i=1}^{n}$ matching the interior landmarks, which is the unique extremal extension of $g$ to $\mathbb{D}$. That is, $f: \mathbb{D} \backslash\left\{p_{i}\right\}_{i=1}^{n} \rightarrow$ $\mathbb{D} \backslash\left\{q_{i}\right\}_{i=1}^{n}$ is an extremal Teichmüller map with $\left.f\right|_{\partial \mathbb{D}}=g$ matching the interior landmarks.

2.3. Harmonic maps. Our iterative algorithm to compute Teichmüller maps is closely related to harmonic maps. Let $\left(S_{1}, \sigma|d z|^{2}\right)$ and $\left(S_{2}, \rho|d w|^{2}\right)$ be two Riemann surfaces of finite type, where $z$ and $w$ refer to the local conformal coordinate on the surface $S_{1}$ and $S_{2}$.

For a Lipschitz map $f:\left(S_{1}, \sigma|d z|^{2}\right) \rightarrow\left(S_{2}, \rho|d w|^{2}\right)$, we define the energy $E(f ; \sigma, \rho)$ of the map $w$ to be

$$
E_{\text {harm }}(f ; \sigma, \rho)=\int_{S_{1}} \frac{1}{2}\|d f\|^{2} d v(\sigma)=\int_{S_{1}} \frac{\rho(w(z))}{\sigma(z)}\left(\left|w_{z}\right|^{2}+\left|w_{\bar{z}}\right|^{2}\right) \sigma(z) d z d \bar{z} .
$$

Therefore

$$
E_{h a r m}(f ; \sigma, \rho)=\int_{S_{1}} \rho(w(z))\left(\left|w_{z}\right|^{2}+\left|w_{\bar{z}}\right|^{2}\right) d z d \bar{z} .
$$

It depends on the metric structure of the target surface $\rho|d w|^{2}$ and the conformal structure $\sigma|d z|^{2}$ of the source.

A critical point of this functional is called a harmonic map. We will focus on the situation where we have fixed the homotopy class $f_{0}: S_{1} \rightarrow S_{2}$ of maps into the compact target $S_{2}$ with non-positive curvature $K(w) \leq 0$ everywhere. In that case, there is a unique harmonic map $f(\sigma, \rho):\left(S_{1}, \sigma\right) \rightarrow\left(S_{2}, \rho\right)$ in the homotopy class of $f_{0}$. If $f$ is harmonic, then

$$
f_{z \bar{z}}+(\log \rho)_{z} f_{z} f_{\bar{z}} \equiv 0 .
$$

The pull back metric on $S_{1}$ induced by $f$ is given by

$$
f^{*}\left(\rho(w)|d w|^{2}\right)=\rho(f(z))\left(\bar{f}_{z} d \bar{z}+\overline{f_{\bar{z}}} d z\right) ;
$$

also, the Hopf differential is

$$
\Phi(f) d z^{2}:=\rho(f(z)) f_{z} \overline{f_{\bar{z}}} d z^{2} .
$$

It can be shown that $f$ is harmonic if and only if its Hopf differential is a holomorphic quadratic differential. 


\section{QUASI-CONFORMAL ITERATION}

Before giving a complete analysis of the convergence of the QC iteration, we reformulate the extremal problem (1.1) as the optimization problem of the harmonic energy, in order to better understand the iterative algorithm.

Consider two connected open surfaces $S_{1}$ and $S_{2}$ with boundaries, which are of the same topology. $S_{1}$ and $S_{2}$ can either be simply-connected or multiply-connected. Suppose $\sigma|d z|^{2}$ and $\rho|d w|^{2}$ are the Riemannian metrics on $S_{1}$ and $S_{2}$ respectively. Assume $\left(S_{2}, \rho|d w|^{2}\right)$ has non-positive Gaussian curvature $K(w)$ everywhere. Let $f: S_{1} \rightarrow S_{2}$ be any quasi-conformal mapping between $S_{1}$ and $S_{2}$. In the homotopic class $[f]$ of $f$, there exists a unique Teichmüller map, $f^{*} . f^{*}$ is also extremal within the homotopic class $[f]$. More specifically, the homotopic class $[f]$ can be defined as

$$
[f]=\left\{g: S_{1} \rightarrow S_{2}:\left.g\right|_{\partial S_{1}}=\left.f\right|_{\partial S_{2}}\right\} .
$$

We have, $\left\|\mu_{f^{*}}\right\|_{\infty} \leq\left\|\mu_{g}\right\|_{\infty}$ for all $g \in[f]$, where $\mu_{f^{*}}$ and $\mu_{g}$ are the Beltrami differentials of $f^{*}$ and $g$ respectively.

Consider the space of all admissible Beltrami differentials on $S_{1}$, which is denoted by $\mathcal{B}\left(S_{1}, S_{2}\right)$. Every Beltrami differential $\mu \in \mathcal{B}\left(S_{1}, S_{2}\right)$ induces a conformal structure $g(\mu)$ on $S_{1}$, namely,

$$
g(\mu)=|d z+\mu d \bar{z}|^{2} .
$$

Suppose $\mu_{1}, \mu_{2} \in \mathcal{B}\left(S_{1}, S_{2}\right)$, we say that they are conformally equivalent, if there is a biholomorphic mapping $f:\left(S_{1}, g\left(\mu_{1}\right)\right) \rightarrow\left(S_{1}, g\left(\mu_{2}\right)\right)$ such that $f$ is homotopic to the identity map of $S_{1}$. The equivalence class of $\mu$ is represented by $[\mu]$. Each conformal equivalence class of Beltrami differentials has a unique representative of Teichmüller form. We denote the space of all Beltrami differentials of constant modulus by

$$
\mathcal{T}\left(S_{1}, S_{2}\right):=\left\{\mu \in \mathcal{B}\left(S_{1}, S_{2}\right):|\mu| \text { is a constant }\right\} .
$$

We will focus on the situation where we have fixed a homotopic class $\left[f_{0}\right]$. We can define an energy functional $E_{B C}$ on $\mathcal{B}\left(S_{1}, S_{2}\right)$. For any $\mu \in \mathcal{B}\left(S_{1}, S_{2}\right)$, there exists a unique harmonic map $f(\mu, \rho):\left(S_{1}, g(\mu)\right) \rightarrow\left(S_{2}, \rho|d w|^{2}\right) \in[f]$, which is solely determined by $\mu$ and $\rho|d w|^{2}$. The value of $E_{B C}(\mu)$ can then be defined as the harmonic energy of $f(\mu, \rho)$. That is,

$$
E_{B C}(\mu)=E_{\text {harm }}(f(\mu, \rho))=\int_{S_{1}} \frac{1}{2}\|d f(\mu, \rho)\|^{2} .
$$

$E_{B C}: \mathcal{B}\left(S_{1}, S_{2}\right) \rightarrow \mathbb{R}$ is a smooth function.

Lemma 3.1. The energy functional $E_{B C}: \mathcal{T}\left(S_{1}, S_{2}\right) \rightarrow \mathbb{R}$ is bounded below by

$$
E_{B C}(\mu) \geq \int_{S_{2}} \rho(w) d u d v
$$

where $w=u+i v$. The equality holds if and only if $\left(S_{1}, g(\mu)\right)$ is conformally equivalent to $\left(S_{2}, \rho|d w|^{2}\right)$; and the harmonic map $f(\mu, \rho):\left(S_{1}, g(\mu)\right) \rightarrow\left(S_{2}, \rho|d w|^{2}\right)$ is a conformal mapping.

Proof. Let $z=x+i y$ be the local coordinate of $\left(S_{1}, g(\mu)\right)$. The Jacobian of the mapping $f(\mu, \rho):\left(S_{1}, g(\mu)\right) \rightarrow\left(S_{2}, \rho|d w|^{2}\right)$ is given by

$$
J(z)=\left|w_{z}\right|^{2}-\left|w_{\bar{z}}\right|^{2} .
$$


Therefore,

$$
J(z) d x d y=\left(\left|w_{z}\right|^{2}-\left|w_{\bar{z}}\right|^{2}\right) d x d y=d u d v .
$$

The harmonic energy is given by

$$
\begin{aligned}
E_{\text {harm }}(f(\mu, \rho))=E_{B C}(\mu) & =\int_{S_{1}} \rho(w)\left(\left|w_{z}\right|^{2}+\left|w_{\bar{z}}\right|^{2}\right) d x d y \\
& =\int_{S_{2}} \rho(w) \frac{\left|w_{z}\right|^{2}+\left|w_{\bar{z}}\right|^{2}}{\left|w_{z}\right|^{2}-\left|w_{\bar{z}}\right|^{2}} d u d v
\end{aligned}
$$

where

$$
\begin{aligned}
\frac{\left|w_{z}\right|^{2}+\left|w_{\bar{z}}\right|^{2}}{\left|w_{z}\right|^{2}-\left|w_{\bar{z}}\right|^{2}} & =\frac{1+\left|\frac{w_{\bar{z}}}{w_{z}}\right|^{2}}{1-\left|\frac{w_{\bar{z}}}{w_{z}}\right|^{2}}=\frac{1+|\mu|^{2}}{1-|\mu|^{2}} \\
& =\frac{1+k^{2}}{1-k^{2}}=\frac{1}{2}\left(\frac{1+k}{1-k}+\frac{1-k}{1+k}\right)=\frac{1}{2}\left(K+\frac{1}{K}\right) \text { and } \\
k & =|\mu|, 0 \leq k<1, K=\frac{1+k}{1-k}, K \geq 1 .
\end{aligned}
$$

Hence,

$$
E_{B C}(\mu)=\frac{1}{2} \int_{S_{2}} \rho(w)\left(K+\frac{1}{K}\right) d u d v \geq \frac{1}{2} \int_{S_{2}} \rho(w)(2) d u d v=\int_{S_{2}} \rho(w) d u d v .
$$

Equality holds if and only if $K \equiv 1$, namely, $k \equiv 0$. This implies $f(\mu, \rho)$ is a conformal mapping.

Theorem 3.2. The global minimizer of the energy functional $E_{B C}: \mathcal{T}\left(S_{1}, S_{2}\right) \rightarrow$ $\mathbb{R}$ is the Beltrami differential associated to the unique Teichmüller map between $\left(S_{1}, \sigma|d z|^{2}\right)$ and $\left(S_{2}, \rho|d w|^{2}\right)$ in the homotopic class $\left[f_{0}\right]$ of $f_{0}$.

Proof. Let $\mu^{*}$ be the Beltrami differential of the Teichmüller map $\tilde{f}$. It suffices to show that $\tilde{f}:\left(S_{1}, g\left(\mu^{*}\right)\right) \rightarrow\left(S_{2}, \rho|d w|^{2}\right)$ is a conformal mapping.

To see this, let $\tilde{f}^{*}\left(\rho|d w|^{2}\right)$ denote the pull back metric. Then,

$$
\tilde{f}^{*}\left(\rho|d w|^{2}\right)=e^{2 \lambda_{2}(\tilde{f}(z))}|d f(z)|^{2} .
$$

Under the pull back metric, the mapping $\tilde{f}:\left(S_{1}, \tilde{f}^{*}\left(\rho|d w|^{2}\right)\right) \rightarrow\left(S_{2}, \rho|d w|^{2}\right)$ is isometric. We have

$$
\begin{aligned}
d \tilde{f}(z) & =\frac{\partial \tilde{f}(z)}{\partial z} d z+\frac{\partial \tilde{f}(z)}{\partial \bar{z}} d \bar{z} \\
& =\frac{\partial \tilde{f}(z)}{\partial z}\left(d z+\mu^{*} d \bar{z}\right) .
\end{aligned}
$$

Hence,

$$
\tilde{f}^{*}\left(\rho|d w|^{2}\right)=e^{2 \lambda_{2}(\tilde{f}(z))}\left|\frac{\partial \tilde{f}(z)}{\partial z}\right|^{2}\left|d z+\mu^{*} d \bar{z}\right|^{2} .
$$

So, $\tilde{f}^{*}\left(\rho|d w|^{2}\right)=e^{2 \lambda_{2}(\tilde{f}(z))}\left|\frac{\partial \tilde{f}(z)}{\partial z}\right|^{2} g\left(\mu^{*}\right) . f^{*}\left(\rho|d w|^{2}\right)$ is conformal to $g\left(\mu^{*}\right)$. We conclude that $\tilde{f}:\left(S_{1}, g\left(\mu^{*}\right)\right) \rightarrow\left(S_{2}, \rho|d w|^{2}\right)$ is conformal. According to Theorem 3.1. the Beltrami differential associated to $\tilde{f}$ is the global minimizer of $E_{B C}$ : $\mathcal{T}\left(S_{1}, S_{2}\right) \rightarrow \mathbb{R}$ 
In other words, finding the extremal Teichmüller map, $f^{*}$, is equivalent to minimizing the energy functional $E_{B C}$. During the QC iteration, the Beltrami differential $\mu_{n} \in \mathcal{T}\left(S_{1}, S_{2}\right)$ is iteratively adjusted and a new map is obtained by $f_{n}=\mathbf{L B S}\left(\mu_{n}\right)$. It turns out $\mathbf{L B S}\left(\mu_{n}\right)$ is equivalent to computing the harmonic map $f\left(\mu_{n}, \rho\right)$. It can be explained in more detail as follows.

Lemma 3.3. Suppose $\mu \in \mathcal{T}\left(S_{1}, S_{2}\right)$. The mapping $f:=\mathbf{L B S}(\mu)$ is a harmonic map between $\left(S_{1}, g(\mu)\right)$ and $\left(S_{2}, \rho|d w|^{2}\right)$.

Proof. Let $\zeta$ be the coordinates of $S_{1}$ with respect to the metric $g(\mu)$. Let $h$ be the harmonic map between $\left(S_{1}, g(\mu)\right)$ and $\left(S_{2}, \rho|d w|^{2}\right)$. Then $h$ is a critical point of the following harmonic energy:

$$
E_{h a r m}(h)=\int_{S_{1}} \rho(h(\zeta))\left(\left|h_{\zeta}\right|^{2}+\left|h_{\bar{\zeta}}\right|^{2}\right) d x d y .
$$

Since $f:=\mathbf{L B S}(\mu)$, according to the definition, $f$ is the critical point of the following energy functional:

$$
E_{L B S}(f)=\int_{S_{1}} \rho(f(z))\left(\left|f_{\bar{z}}-\mu f_{z}\right|^{2}\right) d x d y .
$$

We will show that the above two energy functionals have the same set of critical points.

Note that $d \zeta=d z+\mu d \bar{z}$, then

$$
d \bar{\zeta}=d \bar{z}+\bar{\mu} d z
$$

We obtain

$$
d z=\frac{1}{1-|\mu|^{2}}(d \zeta-\mu d \bar{\zeta}), \quad d \bar{z}=\frac{1}{1-|\mu|^{2}}(-\bar{\mu} d \zeta+d \bar{\zeta})
$$

Hence,

$$
d z \wedge d \bar{z}=\frac{1}{1-|\mu|^{2}} d \zeta \wedge d \bar{\zeta}, \quad h_{\bar{\zeta}}=\frac{1}{1-|\mu|^{2}}\left(h_{\bar{z}}-\mu h_{z}\right) .
$$

Now, the Jacobian $J_{h}$ of $h$ and the Jacobian $J_{f}$ of $f$ are given by

$$
J_{h}=\left|h_{\zeta}\right|^{2}-\left|h_{\bar{\zeta}}\right|^{2}, \quad J_{f}=\left|f_{z}\right|^{2}-\left|f_{\bar{z}}\right|^{2} .
$$

Hence,

$$
\begin{aligned}
& E_{\text {harm }}(h)=\int_{S_{1}} \rho(h(\zeta))\left(2\left|h_{\bar{\zeta}}\right|^{2}+J_{h}\right) i d \zeta \wedge d \bar{\zeta} \\
& =\int_{S_{1}} \rho(h(\zeta))\left[\frac{2}{\left(1-|\mu|^{2}\right)^{2}}\left|h_{\bar{z}}-\mu h_{z}\right|^{2}\right]\left[i\left(1-|\mu|^{2}\right)\right] d z \wedge d \bar{z} \\
& =\int_{S_{1}} \frac{2}{1-|\mu|^{2}} \rho(h(z))\left|h_{\bar{z}}-\mu h_{z}\right|^{2} i d z \wedge d \bar{z}+\int_{S_{1}} \rho(h(\zeta)) J_{h} i d \zeta \wedge d \bar{\zeta} .
\end{aligned}
$$

Since $\mu \in \mathcal{T}\left(S_{1}, S_{2}\right),|\mu|$ is a constant. Thus,

$$
E_{h a r m}(h)=\frac{2}{1-|\mu|^{2}} \int_{S_{1}} \rho(h(z))\left|h_{\bar{z}}-\mu h_{z}\right|^{2} i d z \wedge d \bar{z}+A,
$$

where $A$ is the surface area of $S_{2}$. We conclude that $E_{\text {harm }}$ and $E_{L B S}$ has the same set of critical points. Since $f$ is a critical point of $E_{L B S}, f$ is also a critical point of $E_{\text {harm }}$. Hence, $f$ is a harmonic map between $\left(S_{1}, g(\mu)\right)$ and $\left(S_{2}, \rho|d w|^{2}\right)$. 
The Beltrami differential $\mu_{n} \in \mathcal{T}\left(S_{1}, S_{2}\right)$ is iteratively adjusted during the QC iteration. In the next section, we will prove that $E_{B C}\left(\mu_{n}\right)$ monotonically decreases to the global minimizer of $E_{B C}$.

\section{Proof of Convergence}

In this section, we prove the convergence of the Quasi-conformal iteration to the desired Teichmüller map.

Lemma 4.1. Suppose $\mu \in \mathcal{B}\left(S_{1}, S_{2}\right)$ is deformed by

$$
\mu \rightarrow \mu+\epsilon \nu \in \mathcal{B}\left(S_{1}, S_{2}\right) .
$$

Then, the variation of $E_{B C}$ satisfies

$$
E_{B C}(\mu+\epsilon \nu) \leq E_{B C}(\mu)-4 \operatorname{Re} \int_{S_{1}} \epsilon \Phi(f(\mu, \rho)) \nu \frac{d z_{\mu} \wedge d \bar{z}_{\mu}}{-2 i}+\mathcal{O}\left(\epsilon^{2}\right),
$$

where $z_{\mu}$ is the coordinates of $S_{1}$ under the metric $g(\mu)$.

Proof. Let $\zeta$ be the coordinate of $S_{1}$ under the metric $g(\mu+\epsilon \nu)$. For simplicity, let $z=z_{\mu}$. Then, we have

$$
d z=d \zeta-\epsilon \nu d \bar{\zeta} ; \quad d \bar{z}=d \bar{\zeta}-\epsilon \bar{\nu} d \zeta
$$

The area element with respect to $z$ is given by

$$
d z \wedge d \bar{z}=d \zeta \wedge d \bar{\zeta}-\epsilon \nu d \bar{\zeta} \wedge d \bar{\zeta}-\epsilon \bar{\nu} d \zeta \wedge d \zeta+\epsilon^{2}|\nu|^{2} d \bar{\zeta} d \zeta
$$

Hence,

$$
d z \wedge d \bar{z}=d \zeta \wedge d \bar{\zeta}+\epsilon^{2}|\nu|^{2} d \bar{\zeta} d \zeta
$$

Similarly,

$$
d \zeta \wedge d \bar{\zeta}=d z \wedge d \bar{z}+\epsilon^{2}|\nu|^{2} d \bar{z} d z .
$$

Let $w=f(\mu, \rho)$. Then,

$$
\begin{aligned}
d w & =w_{\zeta} d \zeta+w_{\bar{\zeta}} d \bar{\zeta} \\
& =w_{z} d z+w_{\bar{z}} d \bar{z} \\
& =w_{z}(d \zeta-\epsilon \nu d \bar{\zeta})+w_{\bar{z}}(d \bar{\zeta}-\epsilon \bar{\nu} d \zeta) \\
& =\left(w_{z}-\epsilon \bar{\nu} w_{\bar{z}}\right) d \zeta+\left(w_{\bar{z}}-\epsilon \nu w_{z}\right) d \bar{\zeta}
\end{aligned}
$$

We get that

$$
w_{\zeta}=w_{z}-\epsilon \bar{\nu} w_{\bar{z}} ; \quad w_{\bar{\zeta}}=w_{\bar{z}}-\epsilon \nu w_{z}
$$

Therefore,

$$
\begin{aligned}
\left|w_{\zeta}\right|^{2}=w_{\zeta} \overline{w_{\zeta}} & =\left(w_{z}-\epsilon \bar{\nu} w_{\bar{z}}\right)\left(\overline{w_{z}}-\epsilon \nu \overline{w_{\bar{z}}}\right) \\
& =\left|w_{z}\right|^{2}+\epsilon^{2}|\nu|^{2}\left|w_{\bar{z}}\right|^{2}-\epsilon \nu w_{z} \overline{w_{\bar{z}}}-\epsilon \bar{\nu} \overline{w_{z}} w_{\bar{z}}
\end{aligned}
$$

Similarly,

$$
\begin{aligned}
\left|w_{\bar{\zeta}}\right|^{2}=w_{\bar{\zeta}} \overline{w_{\bar{\zeta}}} & =\left(w_{\bar{z}}-\epsilon \nu w_{z}\right)\left(\overline{w_{\bar{z}}}-\epsilon \bar{\nu} \overline{w_{z}}\right) \\
& =\left|w_{\bar{z}}\right|^{2}+\epsilon^{2}|\nu|^{2}\left|w_{z}\right|^{2}-\epsilon \nu w_{\bar{z}} \overline{w_{\bar{z}}}-\epsilon \bar{\nu} \overline{w_{z}} w_{\bar{z}} .
\end{aligned}
$$


As a result, we get

$$
\begin{aligned}
& E_{B C}(\mu+\epsilon \nu) \leq E_{\text {harm }}(w)=\int_{S_{1}} \rho(w(\zeta))\left(\left|w_{\zeta}\right|^{2}+\left|w_{\bar{\zeta}}\right|^{2}\right) \frac{d \zeta \wedge d \bar{\zeta}}{-2 i} \\
& =\int_{S_{1}} \rho(w(z))\left(\left|w_{z}\right|^{2}+\left|w_{\bar{z}}\right|^{2}\right) \frac{d z \wedge d \bar{z}}{-2 i}-4 \operatorname{Re} \int_{S_{1}} \epsilon \rho(w(z)) w_{z} \overline{w_{\bar{z}}} \nu \frac{d z \wedge d \bar{z}}{-2 i}+\mathcal{O}\left(\epsilon^{2}\right) \\
& =E_{B C}(\mu)-4 \operatorname{Re} \int_{S_{1}} \epsilon \rho(w(z)) w_{z} \overline{w_{\bar{z}}} \nu \frac{d z \wedge d \bar{z}}{-2 i}+\mathcal{O}\left(\epsilon^{2}\right) \\
& =E_{B C}(\mu)-4 \operatorname{Re} \int_{S_{1}} \epsilon \Phi(f(\mu, \rho)) \nu \frac{d z_{\mu} \wedge d \bar{z}_{\mu}}{-2 i}+\mathcal{O}\left(\epsilon^{2}\right) .
\end{aligned}
$$

This completes the proof of the inequality.

Theorem 4.2. Suppose $\mu \in \mathcal{T}\left(S_{1}, S_{2}\right)$. For any $\alpha>0$, there exists $w \in \mathcal{B}\left(S_{1}, S_{2}\right)$ and $\epsilon: S_{1} \rightarrow \mathbb{R}$ such that

(i) $\mu+\epsilon w \in \mathcal{T}\left(S_{1}, S_{2}\right)$;

(ii) $|\epsilon(p) w(p)|<\alpha$ and $|w(p)|=\mid \mu(f(\mu, \rho))$ for all $p \in S_{1}$, where $\mu(f(\mu, \rho))$ denotes the Beltrami differential of $f(\mu, \rho)$ under the auxiliary metric with respect to $z_{\mu}$;

(iii) $\int_{S_{1}} \epsilon w \Phi(f(\mu, \rho)) \frac{d z_{\mu} \wedge d \bar{z}_{\mu}}{-2 i} \geq 0$.

Proof. Let $\tilde{k}=|\mu|$ and $\nu=\mu(f(\mu, \rho))$. For simplicity, we denote $z:=z_{\mu}$ and $f=f(\mu, \rho)$. Pick $\beta \in \mathbb{R}^{+}$such that

$$
\beta \sup _{p \in S_{1}}|\nu(p)|<\alpha / 4
$$

Consider $\tilde{\mu}=\mu+\beta \nu$.

Suppose

$$
\begin{aligned}
& \Omega_{1}=\left\{p \in S_{1}: \arg (\nu)=\arg (\mu)\right\}, \\
& \Omega_{2}=\left\{p \in S_{1}: \arg (\nu)=-\arg (\mu)\right\} .
\end{aligned}
$$

Let

$$
\gamma=\int_{\Omega_{1}}|\nu| \rho(f(z))\left|f_{z}\right|^{2} \frac{d z_{\mu} \wedge d \bar{z}_{\mu}}{-2 i}-\int_{\Omega_{2}}|\nu| \rho(f(z))\left|f_{z}\right|^{2} \frac{d z_{\mu} \wedge d \bar{z}_{\mu}}{-2 i}
$$

If $\gamma>0$, choose $k$ such that $\tilde{k}<k<\tilde{k}+\alpha / 4$.

If $\gamma<0$, choose $k$ such that $\tilde{k}-\alpha / 4<k<\tilde{k}$.

If $\gamma=0$, choose $k=\tilde{k}$ (including the case when $\Omega_{1}=\Omega_{2}=\emptyset$ ).

Let

$$
r=k \frac{\tilde{\mu}}{|\tilde{\mu}|} ; \quad w=\frac{r-\mu}{|r-\mu|}|\nu| \text { and } \epsilon=\frac{|r-\mu|}{|\nu|} .
$$

By definition, $\mu+\epsilon w=r=k \frac{\tilde{\mu}}{|\tilde{\mu}|} \in \mathcal{T}\left(S_{1}, S_{2}\right)$. Hence, (i) is satisfied.

Now,

$$
|w(p)|=|\nu(p)|=|\mu(f(\mu, \rho))(p)| \text { for all } p \in S_{1} .
$$


Also,

$$
\begin{aligned}
|\epsilon(p) w(p)| & =|r-\mu| \\
& \leq|r-\tilde{\mu}|+|\tilde{\mu}-\mu| \\
& =|r-\tilde{\mu}|+|\beta \nu| \\
& <\frac{3 \alpha}{4}+\frac{\alpha}{4}=\alpha .
\end{aligned}
$$

Thus, (ii) is also satisfied.

Finally, it is easy to check that

$$
\begin{aligned}
& \int_{S_{1} \backslash\left(\Omega_{1} \cup \Omega_{2}\right)} \epsilon w \Phi(f(\mu, \rho)) \frac{d z_{\mu} \wedge d \bar{z}_{\mu}}{-2 i} \\
& =\int_{S_{1} \backslash\left(\Omega_{1} \cup \Omega_{2}\right)} \epsilon w \rho(f(z)) \bar{f}_{\bar{z}} f_{z} \frac{d z_{\mu} \wedge d \bar{z}_{\mu}}{-2 i} \geq 0 .
\end{aligned}
$$

Now, if $\gamma>0$,

$$
\begin{aligned}
& \int_{\Omega_{1}} \epsilon w \Phi(f(\mu, \rho)) \frac{d z_{\mu} \wedge d \bar{z}_{\mu}}{-2 i}+\int_{\Omega_{2}} \epsilon w \Phi(f(\mu, \rho)) \frac{d z_{\mu} \wedge d \bar{z}_{\mu}}{-2 i} \\
= & \int_{\Omega_{1}}(k-\tilde{k})|\nu| \rho(f(z))\left|f_{z}\right|^{2} \frac{d z_{\mu} \wedge d \bar{z}_{\mu}}{-2 i}-\int_{\Omega_{2}}(k-\tilde{k})|\nu| \rho(f(z))\left|f_{z}\right|^{2} \frac{d z_{\mu} \wedge d \bar{z}_{\mu}}{-2 i} \\
= & (k-\tilde{k}) \gamma>0 .
\end{aligned}
$$

If $\gamma<0$,

$$
\begin{aligned}
\int_{\Omega_{1}} & \epsilon w \Phi(f(\mu, \rho)) \frac{d z_{\mu} \wedge d \bar{z}_{\mu}}{-2 i}+\int_{\Omega_{2}} \epsilon w \Phi(f(\mu, \rho)) \frac{d z_{\mu} \wedge d \bar{z}_{\mu}}{-2 i} \\
= & -\int_{\Omega_{1}}(\tilde{k}-k)|\nu| \rho(f(z))\left|f_{z}\right|^{2} \frac{d z_{\mu} \wedge d \bar{z}_{\mu}}{-2 i} \\
& +\int_{\Omega_{2}}(\tilde{k}-k)|\nu| \rho(f(z))\left|f_{z}\right|^{2} \frac{d z_{\mu} \wedge d \bar{z}_{\mu}}{-2 i} \\
= & -(\tilde{k}-k) \gamma>0 .
\end{aligned}
$$

We conclude that $\int_{S_{1}} \epsilon w \Phi(f(\mu, \rho)) \frac{d z_{\mu} \wedge d \bar{z}_{\mu}}{-2 i} \geq 0$ and hence (iii) is satisfied.

We can now proceed to prove the convergence of the quasi-conformal iteration.

Theorem 4.3. Suppose $S_{1}$ and $S_{2}$ are open Riemann surfaces with boundaries of the same topology. Given a smooth boundary correspondence $h: \partial S_{1} \rightarrow \partial S_{2}$, the quasi-conformal $(Q C)$ iteration (1.3) converges to the unique extremal map, which is also a Teichmüller map.

Proof. Suppose the pair $\left(f_{n}, \mu_{n}\right)$ is obtained at the $n^{\text {th }}$ iteration. The QC iteration first computes a new quasi-conformal map by $f_{n+1}=\mathbf{L B S}\left(\mu_{n}\right)$. According to Lemma 3.3, $f_{n+1}$ is a harmonic map between $\left(S_{1}, g\left(\mu_{n}\right)\right)$ and $\left(S_{2}, \rho|d w|^{2}\right)$. A new Beltrami differential $\widetilde{\mu}_{n+1}$ can be computed by $\widetilde{\mu}_{n+1}=\mu_{n}+\beta \mu\left(f_{n+1}, \mu_{n}\right)$. Here, $\mu\left(f_{n+1}, \mu_{n}\right)$ denotes the Beltrami differential of $f_{n+1}$ under the auxiliary metric with respect to $\mu_{n}$, namely, $\left|d z+\mu_{n} d \bar{z}\right|^{2}$. $\widetilde{\mu}_{n+1}$ can then be projected onto $\mathcal{T}\left(S_{1}, S_{2}\right)$ to get

$$
\mathcal{P}\left(\widetilde{\mu}_{n+1}\right)=\mu_{n}+\epsilon w_{n} .
$$




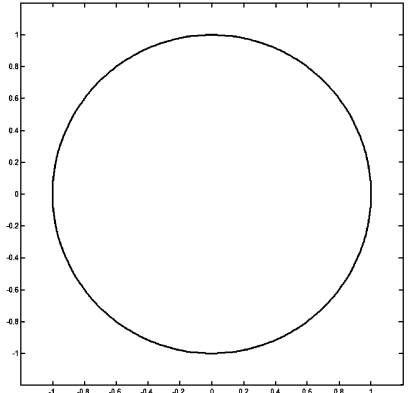

(A)

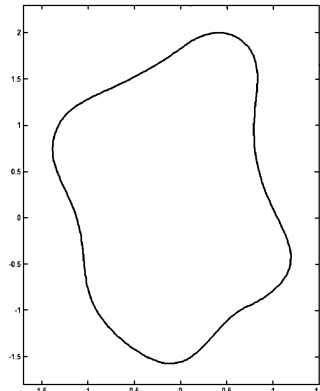

(B)

Figure 3. Two simply-connected domains. (A) a unit disk $\mathbb{D}$;

(B) an arbitrary simply-connected domain.

Here, $w_{n}: S_{1} \rightarrow \mathbb{C}$ and $\epsilon: S_{1} \rightarrow \mathbb{R}$ is a suitable real function on $S_{1}$ such that $\left|\mu_{n}+\epsilon \mu\left(f_{n+1}, \mu_{n}\right)\right| \equiv k$, where $k$ is a positive constant.

According to Theorem 4.2, by choosing a suitable $k$, we can assume that

$$
\int_{S_{1}} \epsilon w_{n} \Phi(f(\mu, \rho)) \frac{d z_{\mu} \wedge d \bar{z}_{\mu}}{-2 i}=\int_{S_{1}} \epsilon\left(\mathcal{P}\left(\widetilde{\mu}_{n+1}\right)-\mu_{n}\right) \Phi(f(\mu, \rho)) \frac{d z_{\mu} \wedge d \bar{z}_{\mu}}{-2 i} \geq 0 .
$$

The Laplacian operator $\mathcal{L}$ is then applied on $\widetilde{\theta}_{n+1}:=\arg \left(\mathcal{P}\left(\widetilde{\mu}_{n+1}\right)\right)$ through

$$
\frac{d \widetilde{\theta}_{n+1}}{d t}=\Delta \widetilde{\theta}_{n+1} .
$$

We choose a suitable diffusion time period $t>0$ to smooth out $\widetilde{\theta}_{n+1}$ such that $\mathcal{L}\left(\mathcal{P}\left(\widetilde{\mu}_{n+1}\right)\right)$ still preserves (4.20). That is,

$$
\int_{S_{1}} \epsilon w_{n}^{\prime} \Phi\left(f(\mu, \rho) \frac{d z_{\mu} \wedge d \bar{z}_{\mu}}{-2 i} \geq 0,\right.
$$

where $w_{n}^{\prime}:=\mathcal{L}\left(\mathcal{P}\left(\widetilde{\mu}_{n+1}\right)\right)-\mu_{n}$.

We get that

$$
E_{B C}\left(\mu_{n+1}\right)-E_{B C}\left(\mu_{n}\right)=-4 \operatorname{Re} \int_{S_{1}} \epsilon w_{n}^{\prime} \Phi\left(f\left(\mu_{n}, \rho\right)\right) \frac{d z_{\mu} \wedge d \bar{z}_{\mu}}{-2 i}+\mathcal{O}\left(\epsilon^{2}\right) \leq 0 .
$$

Hence, $E\left(\mu_{n}\right)$ is monotonically decreasing. According to Lemma 3.1. $E$ is bounded from below. Hence, $E\left(\mu_{n}\right)$ converges. Also, the QC iteration is essentially the gradient descend algorithm of $E_{B C}$ and it converges at the critical point $\mu^{*}=k^{*} e^{i \theta}$. At the critcal point, $\Phi\left(f\left(\mu^{*}, \rho\right)\right)=0$. In this case, $g\left(\mu^{*}\right)$ is conformal to $\rho$ and hence $f\left(\mu^{*}, \rho\right)$ is a quasi-conformal map with Beltrami differential $\mu^{*}$. Furthermore, at the critical point, the Laplacian $\mathcal{L}$ of the argument of the Beltrami differential is zero. We conclude that $\theta$ is harmonic. Since $\theta$ is harmonic, we can find its harmonic conjugate $r$ such that $r-i \theta$ is holomorphic. Define $\varphi=e^{r-i \theta}$, which is also holomorphic. Then, $\mu^{*}=k^{*} \frac{\bar{\varphi}}{|\varphi|}$ is of Teichmüller type. Since $\mu^{*}$ is of Teichmüller type, $f\left(\mu^{*}, \rho\right)$ must be a Teichmüller map. Now, given a smooth boundary correspondence $h: \partial S_{1} \rightarrow \partial S_{2}$, there exists a unique Teichmüller map which is an extremal map. We conclude that $f\left(\mu^{*}, \rho\right)$ is the unique extremal Teichmüller map. 


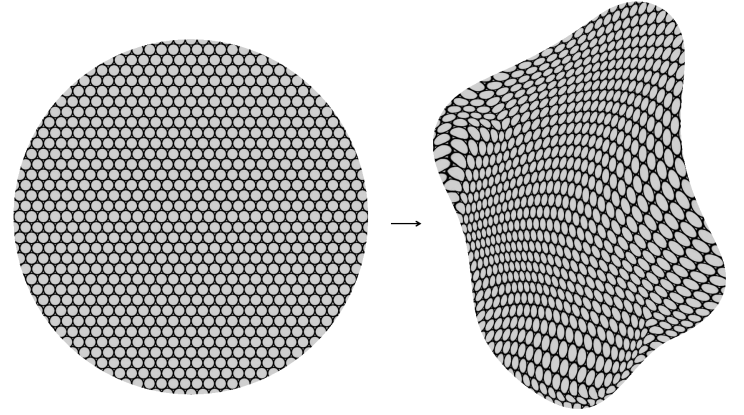

Figure 4. Extremal Teichmüller map between two simplyconnected domains as shown in Figure 3(A) and (B), with given boundary correspondence.

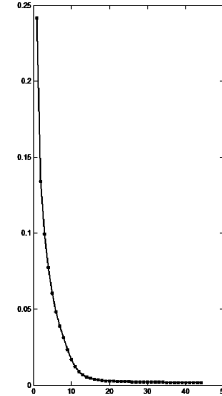

(A)

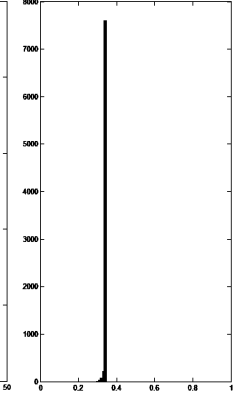

(B)

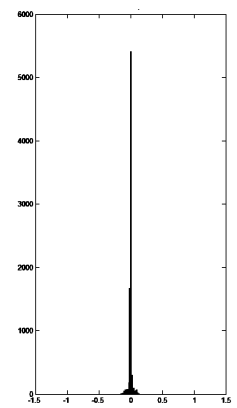

(C)

Figure 5. (A) shows the energy $E\left(\mu_{n}\right):=E_{B C}\left(\mu_{n}\right)-A\left(\Omega_{2}\right)$ per iterations during the QC iterations of Example 1. (B) shows the histogram of the norm of the optimal Beltrami coefficient $\mu^{*}$. (C) shows the histogram of the Laplacian of $\arg \left(\mu^{*}\right)$.

\section{NUMERICAL EXPERIMENTS}

Although the numerical testing is not the main focus of this work, we demonstrate some numerical results in this section for the completeness of the paper. The results agree with our theoretical findings.

Example 1. We first test the algorithm to compute the extremal Teichmüller map between two simply-connected domains $\Omega_{1}$ and $\Omega_{2}$. $\Omega_{1}$ is chosen to be the unit disk $\mathbb{D}$ as shown in Figure 3(A). $\Omega_{1}$ is deformed to an arbitrary simply-connected shape $\Omega_{2}$ as shown in (B). The boundary correspondence $h$ of $\Omega_{1}$ and $\Omega_{2}$ is given. We compute the extremal Teichmüller map $f: \Omega_{1} \rightarrow \Omega_{2}$ such that $\left.f\right|_{\partial \Omega_{1}}=h$ using the proposed QC iterations. The obtained map is visualized using texture map as shown in Figure 4. The small circles on the source domain is mapped to small ellipses on the target domain with the same eccentricity. Figure [5) (A) shows the energy $E\left(\mu_{n}\right):=E_{B C}\left(\mu_{n}\right)-A\left(\Omega_{2}\right)$ versus each of the iterations in the QC iterations, where $A\left(\Omega_{2}\right)$ is the area of $\Omega_{2}$. The energy monotonically decreases to 0 , which agrees with Theorem 4.3 (B) shows the histogram of the norm of the optimal Beltrami differential $\mu^{*}$. It accumulates at 0.33 , which illustrates that the 


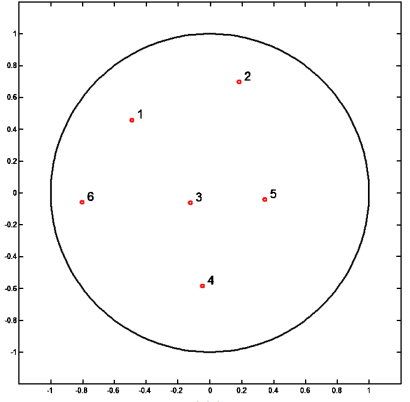

(A)

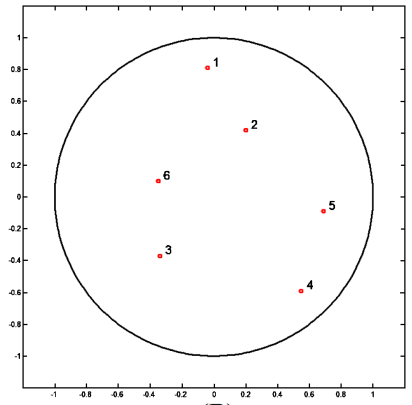

(B)

Figure 6. Two punctured unit disks. (A) and (B) show two unit disks, each with 6 punctures.
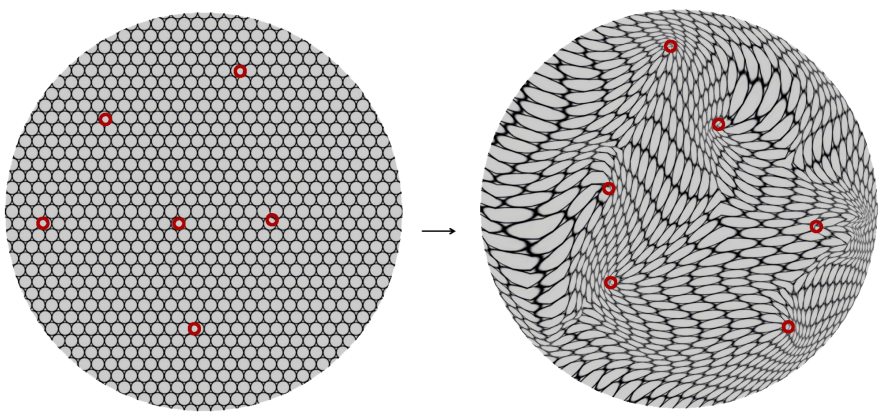

Figure 7. Extremal Teichmüller map between two punctured unit disks as shown in Figure 6(A) and (B), with given boundary correspondence.

obtained map is indeed a Teichmüller map. Since $\mu^{*}$ is of Teichmüller type, its argument must be harmonic. (C) shows the histogram of the Laplacian of $\arg \left(\mu^{*}\right)$. It accumulates at 0 , meaning that the argument of $\mu^{*}$ is indeed harmonic.

Example 2. In our second example, we test our algorithm to compute the extremal Teichmuller map between two punctured unit disks. Figure 6(A) and (B) show two unit disks, each with 6 punctures. Denote the source domain by $\Omega_{1}:=\mathbb{D} \backslash\left\{p_{i}\right\}_{i=1}^{6}$, and denote the target domain by $\Omega_{2}:=\mathbb{D} \backslash\left\{q_{i}\right\}_{i=1}^{6}$. The boundary correspondence of $\partial \mathbb{D}$ is chosen to be the identity map. Using the QC iteration, we compute the extremal Teichmüller map $f: \Omega_{1} \rightarrow \Omega_{2}$ such that $\left.f\right|_{\partial \mathbb{D}}=$ id and $f\left(p_{i}\right)=q_{i}$ for $1 \leq i \leq 6$. The obtained map is visualized using a texture map as shown in Figure 7 The small circles on the source domain is mapped to small ellipses on the target domain with the same eccentricity. Figure 8(A) shows the energy $E\left(\mu_{n}\right):=E_{B C}\left(\mu_{n}\right)-A\left(\Omega_{2}\right)$ versus each of the iterations in the QC iterations, where $A\left(\Omega_{2}\right)$ is the area of $\Omega_{2}$. The energy monotonically decreases to 0 , which agrees with our theoretical finding. (B) shows the histogram of the norm of the Beltrami differential. It accumulates at 0.6 , which illustrates that the obtained map is indeed a Teichmüller map. (C) shows the histogram of the Laplacian of $\arg \left(\mu^{*}\right)$. It accumulates at 0 , meaning that the argument of $\mu^{*}$ is indeed harmonic. 


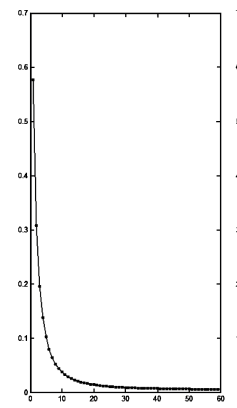

(A)

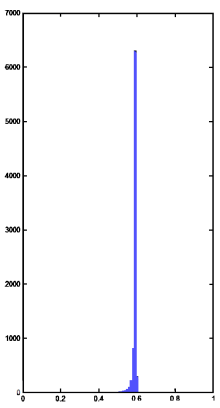

(B)

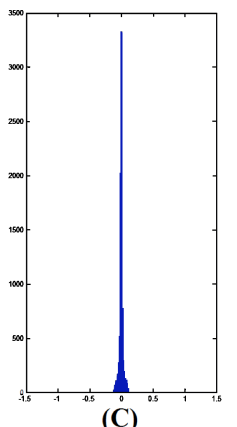

(C)

Figure 8. (A) shows the energy $E\left(\mu_{n}\right):=E_{B C}\left(\mu_{n}\right)-A\left(\Omega_{2}\right)$ per iterations during the $\mathrm{QC}$ iterations of Example 2. (B) shows the histogram of the norm of the optimal Beltrami coefficient $\mu^{*}$. (C) shows the histogram of the Laplacian of $\arg \left(\mu^{*}\right)$.

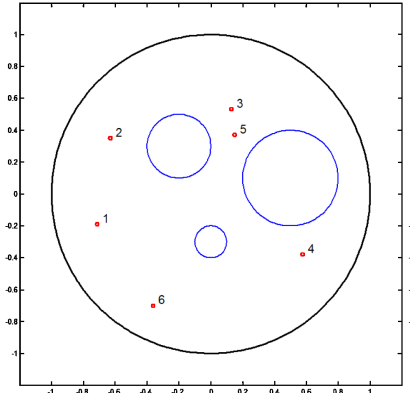

(A)

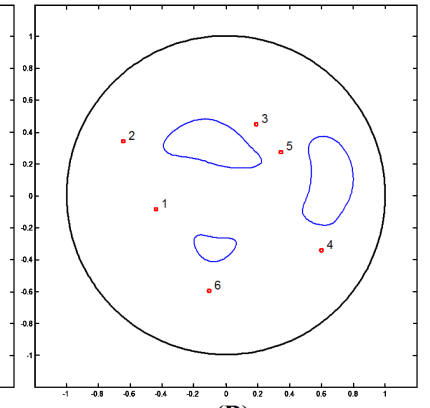

(B)

Figure 9. Two triply-connected domains, each with 6 punctures. (A) and (B) show the source domain and target domain respectively.

Example 3. Finally, we test the QC iterations to compute the extremal Teichmüller map between two triply-connected domains $\Omega_{1}$ and $\Omega_{2}$, each with 6 punctures. As shown in Figure 9(A), $\Omega_{1}$ is chosen to be the unit disk with three inner disks and six points removed (denote it by $\left\{p_{i}\right\}_{i=1}^{6}$ ). $\Omega_{2}$ is chosen to be the unit disk with three inner regions (with arbitrary shapes) and six points removed (denote it by $\left.\left\{q_{i}\right\}_{i=1}^{6}\right)$, as shown in (B). Again, the boundary correspondence $h: \partial \Omega_{1} \rightarrow \partial \Omega_{2}$ is given. Using the QC iterations, we compute the extremal Teichmüller map $f: \Omega_{1} \rightarrow \Omega_{2}$ such that $\left.f\right|_{\partial \Omega_{1}}=h$ and $f\left(p_{i}\right)=q_{i}$ for $1 \leq i \leq 6$. The obtained map is visualized using a texture map as shown in Figure 10, The small circles on the source domain is mapped to small ellipses on the target domain with the same eccentricity. Figure 11(A) shows the energy $E\left(\mu_{n}\right):=E_{B C}\left(\mu_{n}\right)-A\left(\Omega_{2}\right)$ versus each of the iterations in the QC iterations, where $A\left(\Omega_{2}\right)$ is the area of $\Omega_{2}$. The energy monotonically decreases to 0 , which agrees with our theoretical finding. (B) shows the histogram of the norm of the Beltrami differential. It accumulates at 0.42, which illustrates that the obtained map is indeed a Teichmüller map. (C) shows the histogram of the Laplacian of $\arg \left(\mu^{*}\right)$. It accumulates at 0 , meaning that the argument of $\mu^{*}$ is indeed harmonic. 


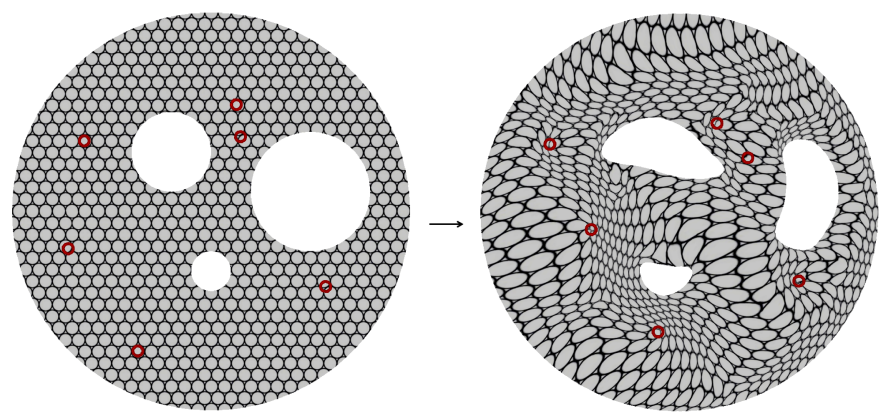

Figure 10. Extremal Teichmüller map between two triplyconnected domains, each with 6 punctures, as shown in Figure 9(A) and (B), with given boundary correspondence.

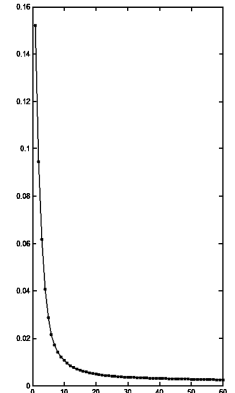

(A)

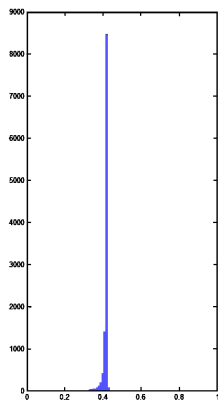

(B)

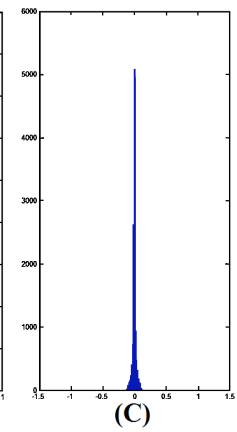

Figure 11. (A) shows the energy $E\left(\mu_{n}\right):=E_{B C}\left(\mu_{n}\right)-A\left(\Omega_{2}\right)$ per iterations during the QC iterations of Example 3. (B) shows the histogram of the norm of the optimal Beltrami coefficient $\mu^{*}$. (C) shows the histogram of the Laplacian of $\arg \left(\mu^{*}\right)$.

\section{Conclusion}

This paper gives the convergence proof of the iterative algorithm proposed in [20] to compute the extremal Teichmüller map between Riemann surfaces of finite type. The iterative algorithm, which is named as quasi-conformal (QC) iteration, can be formulated as the optimization process of the harmonic energy. With this formulation, the QC iteration can be considered as the gradient descent of the harmonic energy under the auxiliary metric given by the Beltrami differentials.

In the future, we will further improve the efficiency of the iterative scheme to optimize the harmonic energy. The proposed framework will also be further extended to compute Teichmüller maps between high-genus surfaces (genus $\geq 1$ ). 


\section{REFERENCES}

[1] A. Fletcher and V. Markovic, Quasiconformal Maps and Teichmüller theory, Oxford Graduate Texts in Mathematics, vol. 11, Oxford University Press, Oxford, 2007. MR2269887 (2007g:30001)

[2] D. G. Crowdy, Conformal slit maps in applied mathematics, Computational Methods and Function Theory 53 (2012), no. 3, 171-189.

[3] D. G. Crowdy, A. S. Fokas, and C. C. Green, Conformal mappings to multiply connected polycircular arc domains, Comput. Methods Funct. Theory 11 (2011), no. 2, 685-706, DOI 10.1007/BF03321882. MR2858967

[4] D. Crowdy and J. Marshall, Conformal mappings between canonical multiply connected domains, Comput. Methods Funct. Theory 6 (2006), no. 1, 59-76, DOI 10.1007/BF03321118. MR.2241034(2007e:30010)

[5] N. Hale and T. W. Tee, Conformal maps to multiply slit domains and applications, SIAM J. Sci. Comput. 31 (2009), no. 4, 3195-3215, DOI 10.1137/080738325. MR 2529786 (2010i:65055)

[6] T. K. Delillo and E. H. Kropf, Numerical computation of the Schwarz-Christoffel transformation for multiply connected domains, SIAM J. Sci. Comput. 33 (2011), no. 3, 1369-1394, DOI 10.1137/100816912. MR2813244

[7] T. K. DeLillo, A. R. Elcrat, E. H. Kropf, and J. A. Pfaltzgraff, Efficient calculation of Schwarz-Christoffel transformations for multiply connected domains using Laurent series, Comput. Methods Funct. Theory 13 (2013), no. 2, 307-336, DOI 10.1007/s40315-013-0023-1. MR3089960

[8] L. N. Trefethen, Numerical computation of the Schwarz-Christoffel transformation, SIAM J. Sci. Statist. Comput. 1 (1980), no. 1, 82-102, DOI 10.1137/0901004. MR.572542(81g:30012a)

[9] T. A. Driscoll and L. N. Trefethen, Schwarz-Christoffel Mapping, Cambridge Monographs on Applied and Computational Mathematics, vol. 8, Cambridge University Press, Cambridge, 2002. MR.1908657 (2003e:30012)

[10] S. Haker, S. Angenent, A. Tannenbaum, R. Kikinis, G. Sapiro, and M. Halle, Conformal surface parameterization for texture mapping, IEEE Transaction of Visualization and Computer Graphics 6 (2000), 181-189.

[11] B. Fischl, M. Sereno, R. Tootell, and A. Dale, High-resolution intersubject averaging and a coordinate system for the cortical surface, Human Brain Mapping 8 (1999), 272-284.

[12] X. Gu, Y. Wang, T. F. Chan, P. M. Thompson, and S.-T. Yau, Genus zero surface conformal mapping and its application to brain, surface mapping, IEEE Transactions on Medical Imaging 23 (2004), no. 8, 949-958.

[13] Y. Wang, L. M. Lui, X. Gu, K. M. Hayashi, T. F. Chan, A. W. Toga, P. M. Thompson, and S.-T. Yau, Brain surface conformal parameterization using riemann surface structure, IEEE Transactions on Medical Imaging 26(2007), no. 6, 853-865.

[14] X. Gu and S.-T. Yau, Computing conformal structures of surfaces, Commun. Inf. Syst. 2 (2002), no. 2, 121-145. MR.1958012 (2003m:65023)

[15] M. K. Hurdal and K. Stephenson, Discrete conformal methods for cortical brain flattening, Neuroimage 45 (2009), 86-98.

[16] R. M. Porter, An interpolating polynomial method for numerical conformal mapping, SIAM J. Sci. Comput. 23 (2001), no. 3, 1027-1041 (electronic), DOI 10.1137/S1064827599355256. MR.1860975 (2002h:30007)

[17] N. Hale and T. W. Tee, Conformal maps to multiply slit domains and applications, SIAM J. Sci. Comput. 31 (2009), no. 4, 3195-3215, DOI 10.1137/080738325. MR2529786 (2010i:65055)

[18] T. K. Delillo and E. H. Kropf, Numerical computation of the Schwarz-Christoffel transformation for multiply connected domains, SIAM J. Sci. Comput. 33 (2011), no. 3, 1369-1394, DOI 10.1137/100816912. MR2813244

[19] F. P. Gardiner and N. Lakic, Quasiconformal Teichmüller theory, Mathematical Surveys and Monographs, vol. 76, American Mathematical Society, Providence, RI, 2000. MR.1730906 (2001d:32016)

[20] L. M. Lui, K. C. Lam, S. T. Yau, X. F. Gu, Teichmüller extremal mapping and its applications to landmark matching registration, (submitted \& under revision), arXiv:1211.2569 (http://arxiv.org/abs/1210.8025) 
[21] O. Lehto and K. I. Virtanen, Quasiconformal Mappings in the Plane, 2nd ed., SpringerVerlag, New York-Heidelberg, 1973. Translated from the German by K. W. Lucas; Die Grundlehren der mathematischen Wissenschaften, Band 126. MR0344463 (49 \#9202)

[22] K. Strebel, On quasiconformal mappings of open Riemann surfaces, Comment. Math. Helv. 53 (1978), no. 3, 301-321, DOI 10.1007/BF02566081. MR.505549 (81i:30041)

[23] E. Reich, Extremal quasiconformal mappings of the disk, Handbook of Complex Analysis: Geometric Function Theory, Vol. 1, North-Holland, Amsterdam, 2002, pp. 75-136, DOI 10.1016/S1874-5709(02)80005-1. MR.1966190(2004c:30036)

Department of Mathematics, The Chinese University of Hong Kong, Shatin, Hong KONG

E-mail address: lmlui@math.cuhk.edu.hk

Department of Computer Sciences, State University of New York at Stony Brook, Stony Brook, New York

E-mail address: gu@cs.sunysb.edu

Department of Mathematics, Harvard University, Cambridge, Massachusetts

E-mail address: yau@math.harvard.edu 